Diffusion-weighted imaging (DWI) is an advanced magnetic resonance imaging technique, based on the analysis of thermallydependent movement of water molecules in the extracellular space. This method allows to examine pathological changes at the cellular level which are not visible on conventional magnetic resonance (MR). Apparent diffusion coefficient (ADC) is a mathematically calculated value indicating the diffusion. Reduced $A D C$ value demonstrates a restricted diffusion while increased ADC value indicates a facilitated diffusion.

Aim: The aim of this study was to assess changes in ADC values within normal appearing white matter (NAWM) and normal appearing gray matter (NAGM) in patients with MS before initiating the treatment with IFN-Beta and already in the course of treatment, in comparison with a control group.

Material and Methods: The study comprised 84 patients divided into three groups: patients with MS before initiating the therapy with IFN-Beta (MS0, $\mathrm{n}=28: 22$ women, 6 men; average age: 28.8), patients with MS after at least one year of treatment with IFN-Beta (MS1, $\mathrm{n}=28: 22$ women, 6 men; average age: 30.2 ) and a control group of patients with no intracranial pathology (CG, $\mathrm{n}=28: 20$ women, 8 men; average age: 29.8 ).

$\mathrm{ADC}$ was measured in 11 regions of interest (ROIs), as follows: 1,2 - cerebellum at the level of dentate nuclei, 3 - pons, 4, 5 thalamus, 6,7 - caudate nuclei, 8,9 - frontal white matter regions, 10,11 - fronto-parietal white matter at the convexity. The results were compared between the groups.

The study was performed using a $1.5 \mathrm{~T}$ GE MRI scanner. DWI sequence was performed with the following parametres: $b=0$ and $\mathrm{b}=1000, \mathrm{TR}=8000, \mathrm{TE}=81.2$, slice thickness $=4 \mathrm{~mm}$

Results: In the examined areas of brain, in ROIs: 8 and 9 the ADC values were statistically significantly higher in MS0 group in comparison with $\mathrm{CG}(\mathrm{p}<0.01)$. There were no significant differences in ADC values in ROIs: 8 and 9 between MS1 and CG. However, in ROI 6 the ADC values were significantly lower $(\mathrm{p}<0.01)$, while in ROIs: 10 and 11 significantly higher $(\mathrm{p}<0.01)$ in MS1 group in comaprison with CG.

No other statistically significant differences were found between groups: MS0 and CG, MS1 and CG, as well as MS0 and MS1.

Conclusions: Increased ADC values in NAWM of the frontal lobes in patients with MS before initiating the treatment with IFN-Beta may indicate early axonopathy, in the course of which diffusion is facilitated. Normalization of ADC values in this region after several months of treatment with IFN-Beta as well as decrease in ADC values in NAGM of right caudate nucleus may indicate a decrease of the diffusion and delay in the axonal damage as a result of immunomodulatory treatment.

\section{P.1.i.021 Improvement of well-being measures after regular physical exercise correlates with resting state connectivity in attentional and default mode networks}

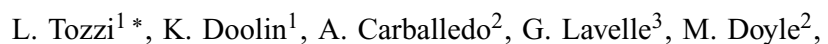
H. McCarthy ${ }^{1}$, V. O'Keane ${ }^{2}$, T. Frodl ${ }^{1}{ }^{1}$ Trinity College Institute of Neuroscience, Psychiatry, Dublin, Ireland; ${ }^{2}$ Trinity College Dublin, Psychiatry, Dublin, Ireland; ${ }^{3}$ Trinity College Dublin, Physiotherapy, Dublin, Ireland

Introduction: Aerobic exercise has been shown to increase performance across several tasks and to exert several effects on the central nervous system detectable by the use of neuroimaging techniques $[1,2]$.
Recently, MRI studies have found that an increased functional connectivity is found after prolonged regular physical exercise in brain networks in the resting state, such as the default mode network (DMN), the fronto-parietal network and in areas of the affective network and that this increase correlates with an improvement of executive function $[3,4]$.

Aims: Our study aimed to assess resting state modifications across known brain networks after an extensive period of physical exercise and to investigate their correlations with behavioural measures of mood state and perceived health.

Methods: Thirty-eight healthy participants were recruited. They were then randomised to either participate to an aerobic exercise programme twice a week for 16 weeks or to be part of a control group.

Physical health was assessed with the RAND 36-Item Health Survey and mood was assessed with the Profile of Mood States questionnaire before the start of the program and at its end. We analysed which scores changed between the first and last timepoint in the exercise group while not changing in the control group through paired t-tests and between groups t-tests $(\mathrm{p}<0.05)$.

Magnetic resonance images from each participant were obtained at $3 \mathrm{~T}$ in the resting state before the start of the exercise programme and after its end. We computed functional connectivity measures by correlating the timeseries of the following seed regions against all other voxels within the brain: anterior cingulate cortex, temporo-parietal junction and ventero-frontal cortex (ventral attention network); inferior parietal sulcus (IPS) and frontal eye fields (FEF) (dorsal attention network); dorsolateral prefrontal cortex (cognitive control network) and precuneus (PCC) (DMN).

A flexible factorial model was used in SPM9 and the brain regions showing a significant interaction between the group and time factors were identified ( $\mathrm{F}$ test, $\mathrm{p}<0.05 \mathrm{FDR}$ ). Post-hoc t-tests were performed to select the regions for which this interaction was driven by a difference between the two timepoints in the exercise group $(\mathrm{p}<0.05$ FDR $)$.

Finally, correlations were investigated between the changes in the questionnaire scores before and after the exercise programme and those in resting state activity in the resulting areas (Spearman test, $\mathrm{p}<0.05$ ).

Results: After the programme, exercise group participants showed a change and better final scores for general health, tension and vigour.

Three pairs of regions changed their connectivity in the exercise group compared to the control group after exercise: $\mathrm{FEF} /$ caudate tail; PCC/superior parietal lobule; IPS/insula.

In all participants, changes in vigour scores correlated with the ones in the connectivity measures between FEF/caudate tail and PCC/superior parietal lobule.

Discussion: Following physical exercise, regions belonging to the attention networks and the DMN change their connectivity with areas involved in emotional processing and visual attention. Increased connectivity between these areas might be related the increased feeling of vigour following the programme.

\section{References}

[1] C.L. Davis, P.D. Tomporowski, J.E. McDowell, B.P. Austin, P.H. Miller N.E. Yanasak, et al., 2011. Exercise Improves Executive Function and Achievement and Alters Brain Activation in Overweight Children: A Randomized Controlled Trial, Health Psychol. Off. J. Div. Health Psychol. Am. Psychol. Assoc. 30, 91-98. doi:10.1037/a0021766.

[2] N. Evero, L.C. Hackett, R.D. Clark, S. Phelan, T.A. Hagobian, 2011. Aerobic exercise reduces neuronal responses in food reward brain regions, J. Appl. Physiol. Bethesda Md 1985. 112 (2012) 1612-1619. doi:10.1152/japplphysiol.01365. 
[3] M.W. Voss, S. Heo, R.S. Prakash, K.I. Erickson, H. Alves, L. Chaddock, et al., 2013. The influence of aerobic fitness on cerebral white matter integrity and cognitive function in older adults: Results of a one-year exercise intervention, Hum. Brain Mapp. 34, 2972-2985. doi:10.1002 hbm.22119.

[4] M.W. Voss, R.S. Prakash, K.I. Erickson, C. Basak, L. Chaddock, J.S Kim, et al., 2010. Plasticity of Brain Networks in a Randomized Intervention Trial of Exercise Training in Older Adults, Front. Aging Neurosci. 2 doi:10.3389/fnagi.2010.00032.

\section{P.1.i.022 Effect of brain-derived neurotrophic factor genotype, gene expression and serum protein levels and childhood maltreatment on regional brain volume}

L.S. Van Velzen ${ }^{1}$, L. Schmaal ${ }^{1}$, R. Jansen ${ }^{1}$, Y. Milaneschi ${ }^{1}$, M.J. Van $\mathrm{Tol}^{2}$, B.M. Elzinga ${ }^{3}$, N.J.A. Van der $\mathrm{Wee}^{3}$, D.J. Veltman ${ }^{1}$, B.W.J.H. Penninx ${ }^{1}{ }^{1} V U$ University Medical Center and GGZ inGeest, Department of Psychiatry and Neuroscience Campus Amsterdam, Amsterdam, The Netherlands; ${ }^{2}$ University Medical Center, Neuroimaging Center, Groningen, The Netherlands; ${ }^{3}$ Leiden University, Institute of Psychology and Leiden Institute for Brain and Cognition, Leiden, The Netherlands

Background: Childhood maltreatment (CM) has been associated with reduced volume of the hippocampus, amygdala and prefrontal cortex [1-3], which may partly be due to a direct impact on neural growth, e.g. through the brain-derived neurotrophic factor (BDNF) pathway. In addition, susceptibility for CM may be genetically determined as previous studies have reported an interaction between $\mathrm{CM}$ and BDNF genotype on brain volume [4,5]. However, findings on $\mathrm{CM}$ and BDNF pathway as determinants of brain volume are inconsistent and have never accounted for the entire BDNF pathway. We examined the effects of CM, the BDNF pathway (at the gene, gene expression and protein level) and their interactions on volume of the hippocampus and amygdala and thickness of the caudal and rostral anterior cingulate cortex (ACC).

Methods: Data were collected from 288 subjects in the NESDA study. CM was assessed using the Nemesis Childhood Trauma Interview. BDNF genotype, gene expression and serum protein levels were determined from blood samples and T1 magnetic resonance images were acquired at 3T. Regional brain volume and thickness were determined using FreeSurfer. Linear regression analyses with brain volume or thickness as dependent variables and BDNF genotype, gene expression and protein levels a independent variables were performed, while correcting for age, sex, education, scansite and intracranial volume.

Results: CM was associated with decreased volume of the amygdala $(p=0.018)$, but not hippocampus or thickness of the ACC. BDNF gene expression was positively associated with amygdala volume $(p=0.040)$. Gene-environment interaction analyses revealed that maltreated carriers of the BDNF met-allele show especially reduced amygdala volume (interaction effect: $\mathrm{p}<0.001$ ), while maltreated individuals with a val/val genotype show increased caudal and rostral ACC thickness (interaction effect: $p=0.003$ and $p=0.034$ respectively). BDNF gene expression was positively correlated with amygdala volume in subjects without a history of CM but this association was absent in subjects with a history of CM. We did not find any interaction effects on hippocampal volume. Abovementioned results were unaltered after an additional correction for SSRI use, smoking, presence of a psychiatric disorder and population structure. Furthermore, posthoc tests revealed that the BDNF genotype and CM interaction effect on amygdala volume and the BDNF gene expression and $\mathrm{CM}$ interaction effect on amygdala volume were independent, indicating that the effect of BDNF genotype on amygdala volume could not be explained by an effect through BDNF gene expression.

Conclusions: $\mathrm{CM}$ and BDNF interact on multiple levels of the BDNF pathway to alter brain volume. Met-carriers appear to be more sensitive to the effects of early life stress on the amygdala, but not due to decreased BDNF gene expression. More fundamental research is needed to examine the mechanisms underlying the effect of maltreatment on brain volume and the role of the BDNF genotype.

\section{References}

[1] Teicher, M.H., Anderson, C.M., Polcari, A., 2012. Childhood maltreatment is associated with reduced volume in the hippocampal subfields CA3, dentate gyrus, and subiculum. Proceedings of the National Academy of Sciences $109,563-572$

[2] Van Harmelen, A.L., van Tol, M.J., van der Wee, N.J.A., Veltman, D.J., Aleman, A., Spinhoven, P., Elzinga, B.M., 2010. Reduced medial prefrontal cortex volume in adults reporting childhood emotional maltreatment. Biological Psychiatry 68, 832-838.

[3] Hanson, J.L., Nacewicz, B.M., Sutterrer, M.J., Cayo, A.A., Schaefer, S., Rudolph, K.D., Davidson, R.J., 2014. Behavioral Problems After Early Life Stress: Contributions of the Hippocampus and Amygdala. Biological Psychiatry, 1-9.

[4] Gerritsen, L., Tendolkar, I., Franke, B., Vasques, A., Kooijnman, S., Buitelaar, J., Rijpkema, M., 2012. BDNF Val66Met genotype modulates the effect of childhood adversity on subgenual anterior cingulate cortex volume in healthy subjects. Molecular Psychiatry 17, 597-603.

[5] Frodl, T., Skokauskas, N., Frey, E.M., Morris, D., Gill, M., Carballedo, A., 2014. BDNF Val66Met genotype interacts with childhood adversity and influences the formation of hippocampal subfields. $\mathrm{Hu}-$ man Brain Mapping 5783, 5776-5783.

\section{P.1.i.023 Self-regulation of the dopaminergic reward system via real time fMRI neurofeedback: a novel treatment approach for cocaine addiction}

M. Kirschner ${ }^{1}{ }^{*}$, P. Stämpfli ${ }^{1}$, M. Hodel ${ }^{1}$, S. Hösli ${ }^{1}$, E. Engeli ${ }^{1}$, L. Hulka ${ }^{1}$, J. Sulzer ${ }^{2}$, E. Seifritz ${ }^{1}$, B.B. Quednow ${ }^{1}$, M. Herdener ${ }^{1}$ ${ }^{1}$ University of Zürich, Department of Psychiatry - Psychotherapy and Psychosomatics, Zürich, Switzerland; ${ }^{2}$ The University of Texas at Austin, Deptartment of Mechanical Engineering, Austin, USA

Introduction: Cocaine addiction is a severe chronical disorder influencing neuroplasticity in the dopaminergic reward circuits [1]. These maladaptive neuronal changes contribute to compulsive drug use and reduced sensitivity to previously rewarding life situations or natural reinforcers [2]. However to date, no effective pharmaceutical or non-pharmaceutical treatment for cocaine addiction has been identified. Recent research in the field of real time fMRI revealed a novel method to self-regulate the dopaminergic reward system, i.e. neural activity in ventral tegmental area and substantia nigra (VTA/SN), with positive mental imagery and enhance imagery-based activation of these reward regions via real time fMRI mediated neurofeedback. Now, we tested this innovative method for the first time in a clinical population to investigate its potential capability as a novel non-invasive treatment strategy. We hypothesized that cocaine addicted patients can improve selfcontrol over dopaminergic reward circuitry. As a consequence sensitivity to natural and alternative reinforcers may be enhanced and drug or drug cue induced sensitivity of the dopaminergic reward system may be reduced.

Methods: So far nine participants with cocaine use higher than $1 \mathrm{~g} /$ week and for the duration of at least 6 month and eight healthy 